\title{
METHODOLOGY FOR MOTION BASE SIMULATION OF CLOSED LOOP SUPERMANEUVERS ON A CENTRIPUGE SIMULATOR
}

\author{
D. W. Repperger, K. McCloskey, J. Frazier, R. Esken, M.Roark* \\ Armstrong Aerospace Medical Research Laboratory \\ Wright Patterson Air Force Base, Ohio 45433-6573 \\ * Raytheon Service Co., Burlington, MA 01803
}

\begin{abstract}
A methodology for designing a closed loop motion mimulation is discussed in this paper. This procedure is applieable to a centrifuge rimulator as well as other types of derices which can produce both motion and visual fields. The key isues of coordinating the motion feld with the visual simulation are diacussed. Both systems are driven by the stick commands of the pilot, thus making it closed loop. The motion fields simulated are based on supermaneuverable flight trajectories where research is needed to investigate how these oupermaneuvers influence the ability of a pilot to perform a mission. The study of supermaneuverability is, indeed, an important ares of research for the Air Force. It has been whown in performance studies that an agile aireraft has a definite combat edge over a nonagile aircref. Also, using agility, a tectical maneuver can be performed expending less overall aircraft energy $[1,2]$.
\end{abstract}

\section{Introduction}

The study of closed loop supermaneuvers on a centrifuge motion aimulator opens up various areas of research in this area. Supermaneuverability refers to unorthodox flight trajectories $[3,4,5,6,7]$ presently being inveatigated with high performance aircraft. It can be accomplished by at least four known methods: using a high angle of attack ( $\alpha>50$ degrees), thrust vectoring, vortex manipulation for control, or possibly using differential defection of stabililsers or canards. Presently most supermaneuvers heve been flown only with 1/7th. aise prototypes [2], but the "Cobra Maneuver" has been demonatrated at airshows by both Soviet and U.S. high performance aircraft [8]. The key insue involved with fying aircraf in these unusual scenarios is the obvious atructurable, controllability, and stability problems that ocenr. The structural problems can easily be solved by performing these meneuvers at low speeds or high altitudes. If this procedure is used, then vulnerability becomes a problem of concern because both apeed and altitude infiuence how these maneuvers can be safely flown from a structures point of view. In [2] a clase of all pitch and roll angles for an F-15 aircraft are defined (based on date from prototypes) for both controllable and stable flight. Recently [9] feedback controller deaign is being investigated to improve controllability utilising thrust vectoring on FA/18 models to expand the envelope of performance of these aircraft.

Other areas of research in supermaneuverability include the quantification of agility via metrics $[10,11,12,13]$. Performance studies $[1,14,15,16]$ have shown a substantial performance advantage when an agile aireraft flies versus an nonagile aireraft. The agile aircraft has, apparently, a quicker reaction time and more opportunity to produce counter offensive. It was interestingly shown in [1] that the agile aircraft can produce the same tactical maneuver with less total aireraft energy expended.

The main issue of these studies involves the effects on pilots as they fiy these maneuvers. Their ability to perform the misaion, may be compromised and various disorientation effects may occur. At the Armstrong Aeroopace Medical Research Laboratory, Wright Patterson Air Force Base, Ohio, a 3 axes centrifuge simulator exists which can be made to replicate the motion fields of the supermaneuvers. The motion field simulation of 3 known oupermaneuvere (The "Herbat's Maneuver", "The Cobra", and the "Yo-Yo Turn for BFM") have already been wimulated and are discussed in further detail in [17]. This paper will address only the issues of closed loop control of the centrifuge simulator during these rimulations of supermaneuverable trajectories. The coordination of the visual field with the motion field are important iasues brought out in this paper. To illustrate the simplest closed loop simulation, it is necessary to firnt discus a basic example to show how to coordinate both the motion field and the visual scene. 


\section{Example 1- The Basic Concept}

To simulate a motion field on a centrifuge simulator that will match up with a visual scene, we consider firat a simple scenario to describe such a problem. Figure (1) illustrates such a simple scenario involving a target aircraft and a chase aircraft. Note both aircraft have the following esumptions:

(1) Both aircraft are at an altitude of 10,000 feet,

(2) Both aircraft have a velocity of $400 \mathrm{mph}$, and

(3) Both aircraf have a constant diatance between them of 2700 feet.

These assumptions can easily be relaxed in the latter examples. With these assumptions, however, this allows a very simple solution to this problem. There are four steps associated with the coordination of the motion and visual field to make the entire system closed loop.

\section{Step 1- Generation of The Motion Field on The Centrifuge simulator}

The first step is based on the realistic motion field that can be generated by the centrifuge. The term $\mathrm{Gs}$ in figure (2) represents that acceleration component down the spine of the subject (a standard definition used in acceleration studies). The onset and offset rates $( \pm$ $.5 \mathrm{Gr} /$ second) in figure (2) are the maximum attainable from the centrifuge aimulator used for this simulation. Figure (3) illustrates how the 3 axes of the centrifuge achieves this motion field by vectoring the cab outward as the arm increases in speed. Thus the vector sum of the gravity vector and the $\Omega^{2} R$ acceleration combine to produce the desired Gs vector which appears down the spine of the subject. Thus the motion field in figure (2) can be accurately represented on the centrifuge. The next step involves the production of the two aircraft ecenario to match the motion field.

Step 2- Making The Target and Chase Aircraft Scenario To Match The Motion Field

With reference to figure (1), it is desired to have the two aircraft scenario identically match the motion field already specified. Figure (4) illustrates the yaw, roll, and pitch axes of the aircraft. Figures $(5 a-b)$ represent actual turn radius versus Gs (centrifugal acceleration) and time and $G$ required to complete a 180 degree turn. Based on the asoumption of $400 \mathrm{mph}$ speed, and a radive of $R_{1}=5400$ feet $\left(2 \mathrm{G}\right.$ turn) and $R_{2}=2700$ feet ( 4 $G$ turn), the scenario can be described as follows: The target aircraft will fly the motion trajectory in figure (2) if starting at time $t_{0}$, it flies the smaller radius for $\mathbf{3 0}$ seconds (one full circle). After it completes the small circle, it transitions to the larger circle in a 4 second in- terval. The target aircraft then fies the larger circle for $1 / 2$ cycle, and then transitions to the small circle. This process is repeated indefinitely. In terms of the body axes of the aircraft, the yow angle is equal to the angular rate of the circle. e.f. for the amall circle this would be $360 / 30=12$ degrees/recond yaw angle. The pitch angle is sero degrees since the altitude is asumed to not change. To compensate for gravity (figure (1)), the $\Omega^{2} R$ term is $2 \mathrm{G}$ but the gravity is always $1 \mathrm{Gs}$. The aircraft rolls $\left[\tan ^{-1}(2 / 1)=03.4\right.$ degrees] to the left. This produces a total Gs (down the spine of the subject) of $\sqrt{2^{2}+1^{2}}=2.24 \mathrm{Gs}$. For the tight ( $4 \mathrm{G}$ turn), the roll angle advances to $\left[\tan ^{-1}(4 / 1)=75.9\right.$ degrees]. The total Gs is $\sqrt{4^{2}+1^{2}}=4.12$ corrected to gravity. The roll transitions between onset $(+.5 \mathrm{Gs} /$ second) and offset $(-.5 \mathrm{Gs} /$ second) can be assumed to change linearly. Figure $(5 a, b, c, d)$ illuatrate the coordination of the motion fielde with the yew, roll, and pitch of the aircraft axes. The descripton of the visual field can now be presented quite briefly.

\section{Step 8- Producing The Visual Scene}

For most standard video graphics packages, the simulation of the visual field now follows straight forwardly. From Figures (6a-d), the roll, pitch, and yaw of the aircraft are known. In figure (1), the opeed, altitude, and inertial frame are known. The diatance between the aireraft is asumed to be constant at 2700 feet. This in all the information necesang to simulate the vimul scene. The last atep for this aimple example is the characterixing of the otick controller to perform true cloned loop reaponse both in a vivual sense and in a motion cense.

\section{Step 4- Charecterising Stick Response}

To be truly elosed loop, the pilot must pull back on the otick to change both the vioual and the motion field aimultaneously. Figure (7) illustrates in the pitch axis (the atick controls yaw motion in this cave), only. Either a displacement or force stick could be used, however, soume the stick output (in one axea is +10 rolto). For the yaw command, the stick gain is set such that +10 volts yields 12 degrees yow/second of a stick gain of 1.2 degrees yaw per eecond is used. This is for the risul scene only. The +10 rolt stick output is then calibrated for the motion field at 10 volts $=.5 \mathrm{G} /$ second or $.05 G /$ volt second. This calibrates the motion field as per the atick commands. Thus as the pilot pulls the stick back, both the vional field changes (closed loop) and the motion field changes (closed loop). Since both motion and vioion are coordinated with each other and the stick commands, the overall simulation is closed loop. It should be noted that if the pilot does not pull 
the stick back to full deflection, he will lose the target on the screen. This is very similar to the real situation. This simple example now can be easily extrapolated to a more practical example.

\section{Example 2- Expansion To A Realiatic Dogfight}

Figure (8) illustrates the expansion of figure (1) into - combat scenario similar to example (1) but with the aircraft not being required to be in constant turns. The calculations of the motion field remain the same; for the visual field, an alternating roll senario results but the terrain path changes constantly with time. Now this procedure can be extended to any closed loop scenario.

\section{Example 3 - Expansion To Any Combat Scenario}

To extend this approach to any aupermaneuver, the motion field of the target aireraft would be broken up into 3 body axis motion fields consisting of roll, pitch and yaw of the aircraft (denoted as $\Omega_{R}(t), \Omega_{P}(t), \Omega_{Y}(t)$ ) By taking a Fourier tranoform of each trajectory, one can first calculate (for the dominant frequency) a response similar to examples 1 and 2 described previously for only the dominant frequency. The procedure is then repeated for the next Fourier component etc. Then using superposition properties, the sum of the components to the motion simulation can be approximated by the sum of the commands to each fourier component. Figure (9) illustrates the Yo-Yo turn for BFM (Basic Fighter Maneuver) as actually simulated from centrifuge data and explained in further detail in [17].

\section{References:}

[1] Lynch, V. H.D., et al., "Tactical Evaluation of The Air-To- Air Combat Effectiveness of Supermaneuverability", WRDC-TR-90-3035, June, 1990.

[2] Gal-Or, B., 1890, "Novel, Post-Stall, ThrustVectored F-15 RPVs. Laboratory and Flight Tests", First Year Report, The Jet Propulsion Laboratory, Technion, IIT.

[3] Cook, W. J., "Turning On A Dime In Midair", US News and World Report, Feb 20, 1989, pp. 66-58.

[4] Schefter, J., "X-31 - Bow They Are Inventing A Radical New Way To Fly", Popular Science, Feb., 1989, pp. 59-64.
[5] Clark, G., "U.S., West German Firms Team Up To Develop Highly Maneuverable Fighter", Defense News, Aug. 31, 1087, p. 10

[6] Sweetman, B., "Fighter Agility - The 'Bruce Lee Factor"', International Derense Review, vol. 4, 1990, pp. 395-397

[7] Cord, T. and C. Suchomel, "Supermaneuverability", AIAA 84-2386, Nov., 1980.

[8] Aviation Week and Space Technology, "British Pilot Lauds Performance of SU-27", Sept. 24, 1990, pp. 35-40

[9] Chiang, R. Y., M. G. Safonov, K. P. Maden, and J. A. Tekawy, "A Fixed $H^{\infty}$ Controller For A Supermaneuverable Fighter Performing The Herbst's Maneuver ${ }^{n}$, Proceeding of The 1900 IEEE Conference on Decision and Control, Dec., 1990

[10] Dorn, M., "Aircraft Agility: The Science and The Opportunity", AIAA/AHS/ASEE Aircreft Design, Systems, and Operations Conference, August, 1989.

[11] Bitten, R., "Qualitative and Quantitative Comparison of Government and Industry Agility Metrics", AlAA Atmospheric Flight Mechanics Conference, Aug., 1989.

[12] Foltyn, R. W., et al., "Development of Innovative Air Combat Measures of Merit For Supermsneuverable Fighters", AFWAL-TR-87-3073, October, 1987.

[13] Hamilton, W. and A. Skow, "Operational Utility Survey, Supermaneuverability", AFWAL-TR-84-3020, 1984.

[14] Hague, D.S., "Multiple-Tactical Aireraft Combat Performance Evaluation System", J. Aircraft, vol. 18, No. 7, July, 1981, pp. 513-519.

[15] Hague, D. S., "Correlation of Flight Test and Analytic M-on-N Air Combat Exchange Ratios", Journel of Airereft, rol. 20, no. 10, Oet., 1983

[16] Mitchell, A., T. Halley and J. Roedper, "Simulation Analysis: Unorthodox Control Force Fighter Aircraft ${ }^{n}$, AFWAL-TR-80-3060, vol. I, 1980.

[17] Repperger, D.W., "A Study of Supermaneuverable Flight Trajectories Through Motion Field Simulation of A Centrifuge Simulator", under review. 


\section{Figure (1)}

\section{A Simple 2 Aircraft}

Chase Scenario
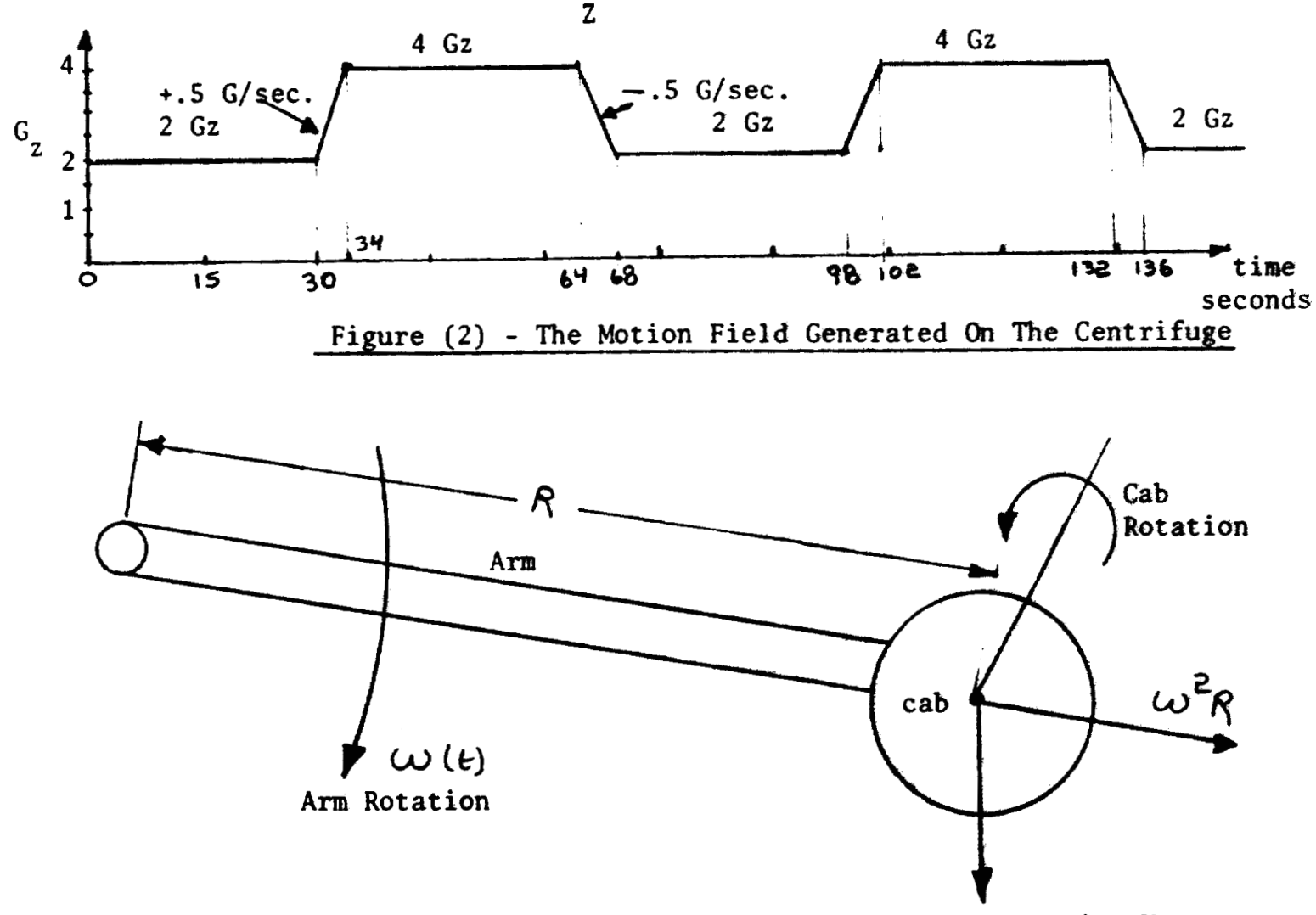

Figure (3) - Principal of Operation of The Centrifuge

1 G Gravity Vector 


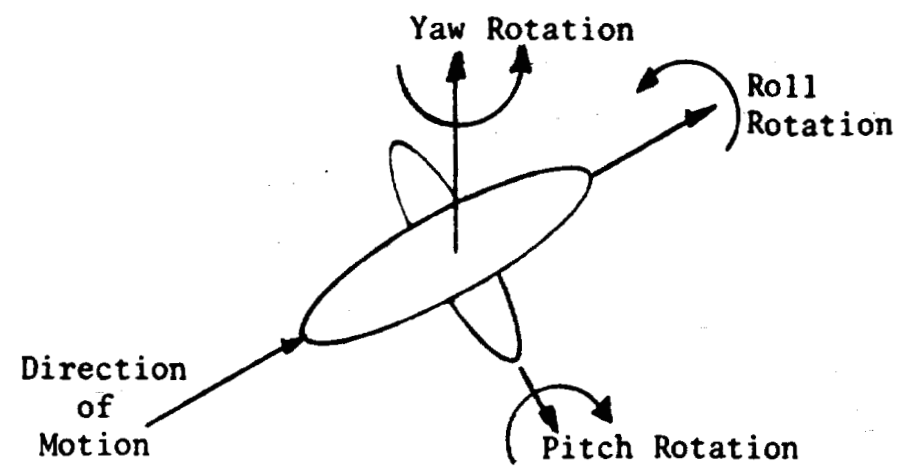

Figure (4) - Yaw, Roll, and Pitch Axes of An Aircraft
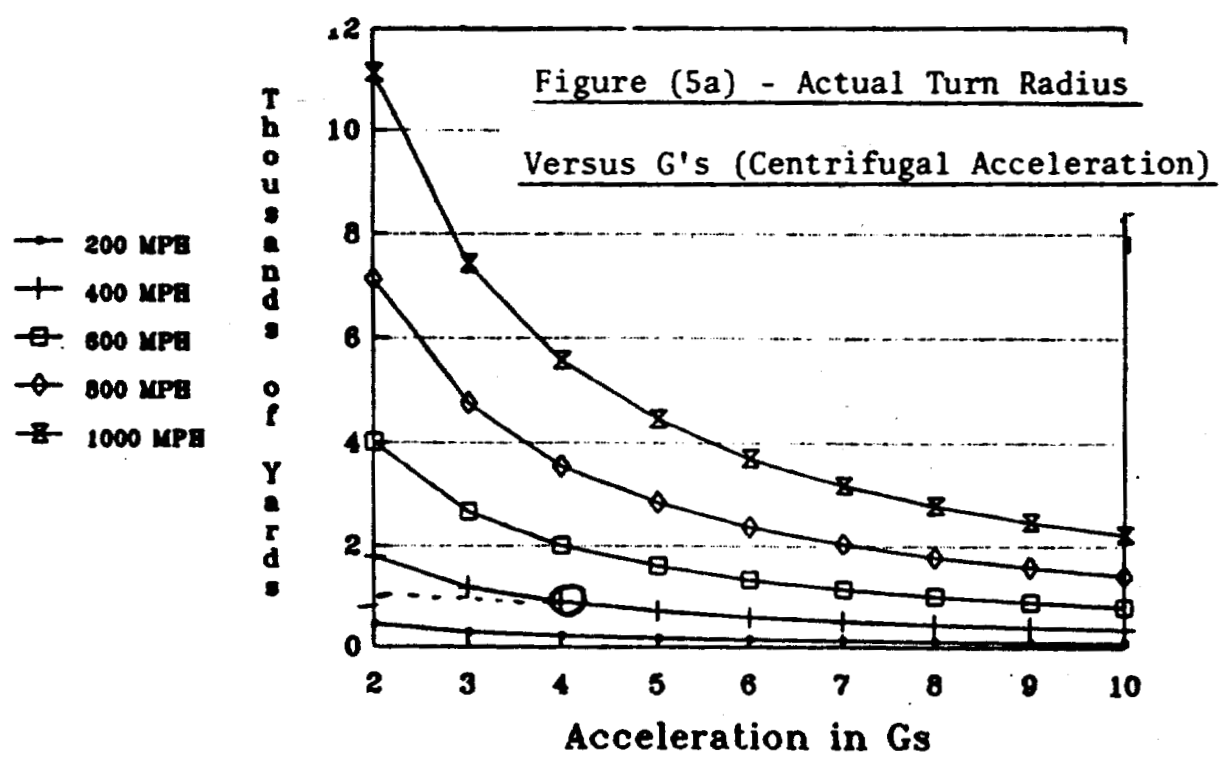

Figure (5b) - Time and G Required To Complete a 180 Degree Turn

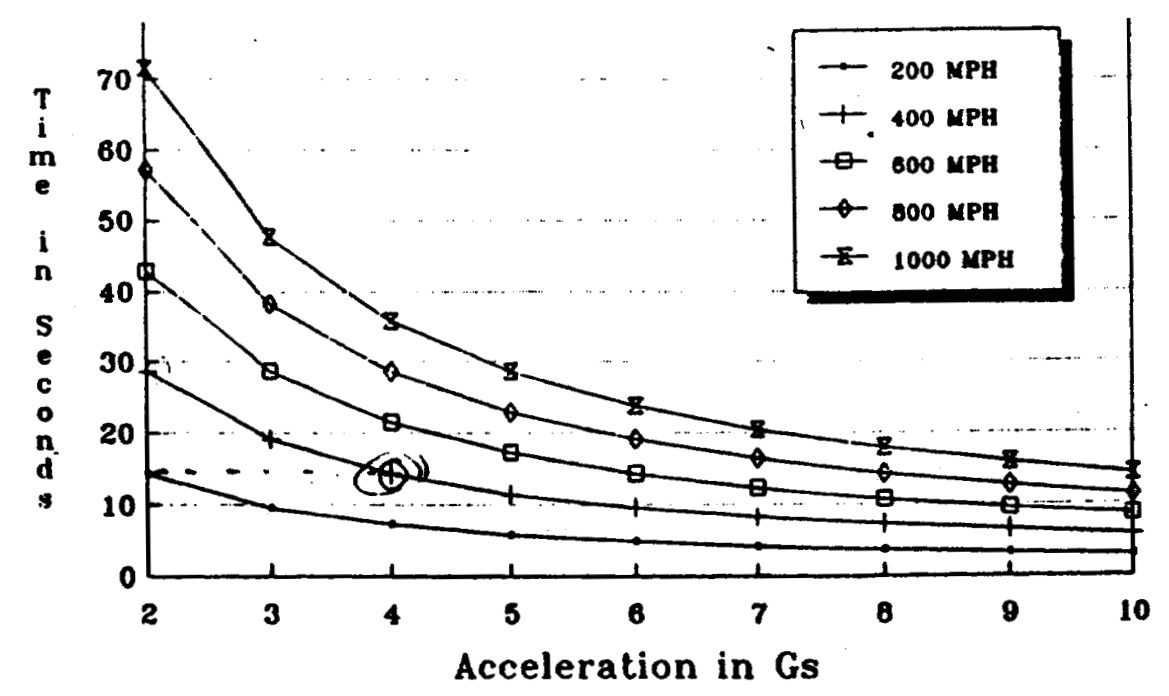



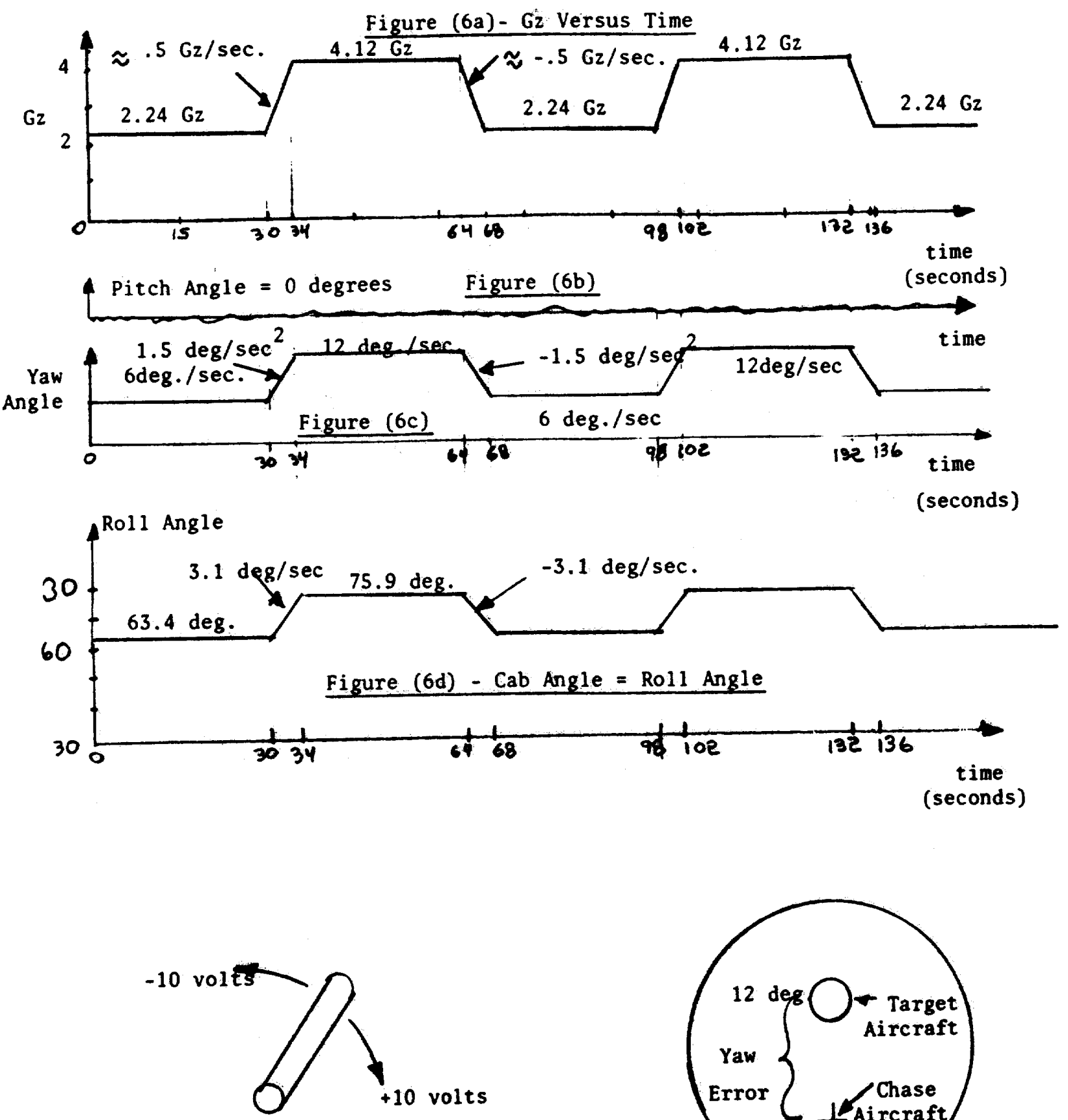

Displacement Stick

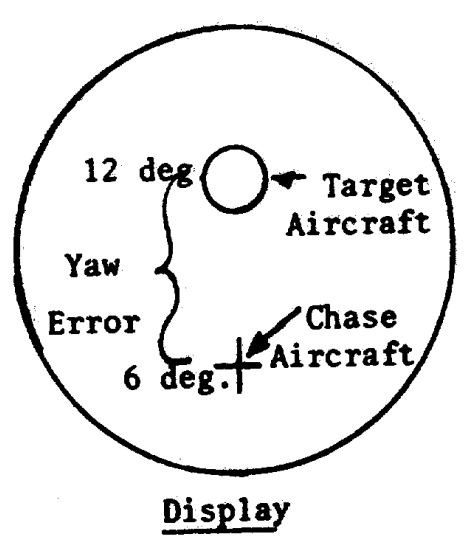

Figure (7)-Displacement Stick and Display 


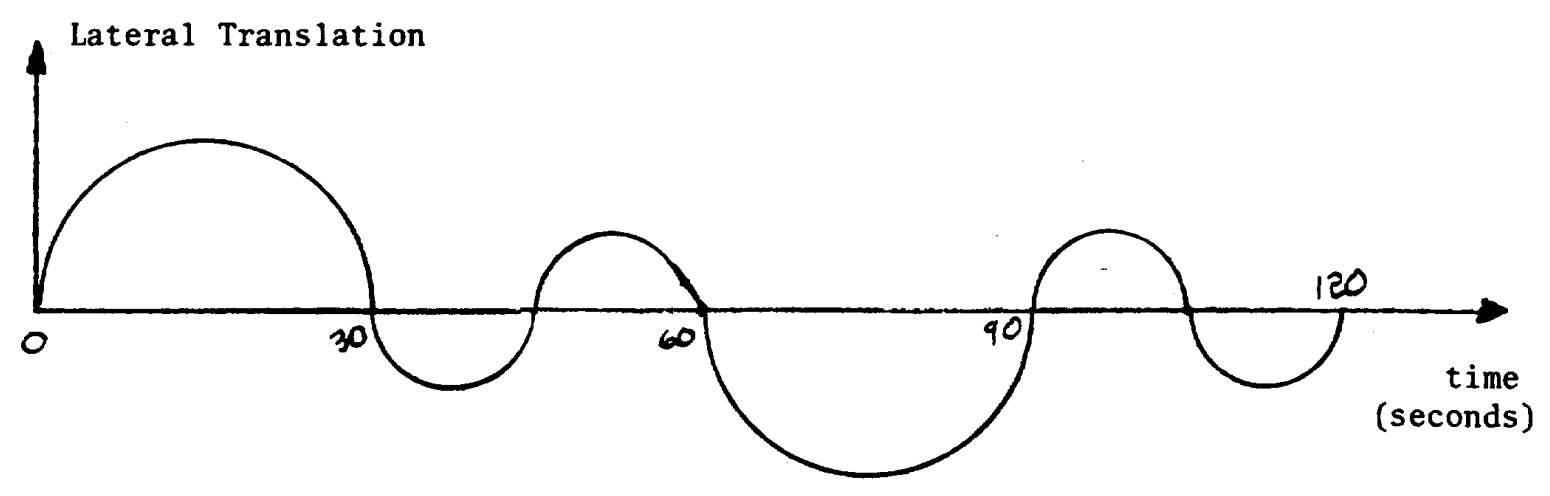

Figure (8) - Lateral Translation For Example 2

Cab and Fork Cammands

Empirical Data

\section{Yo-Yo Turn Simulation on Centrifuge}
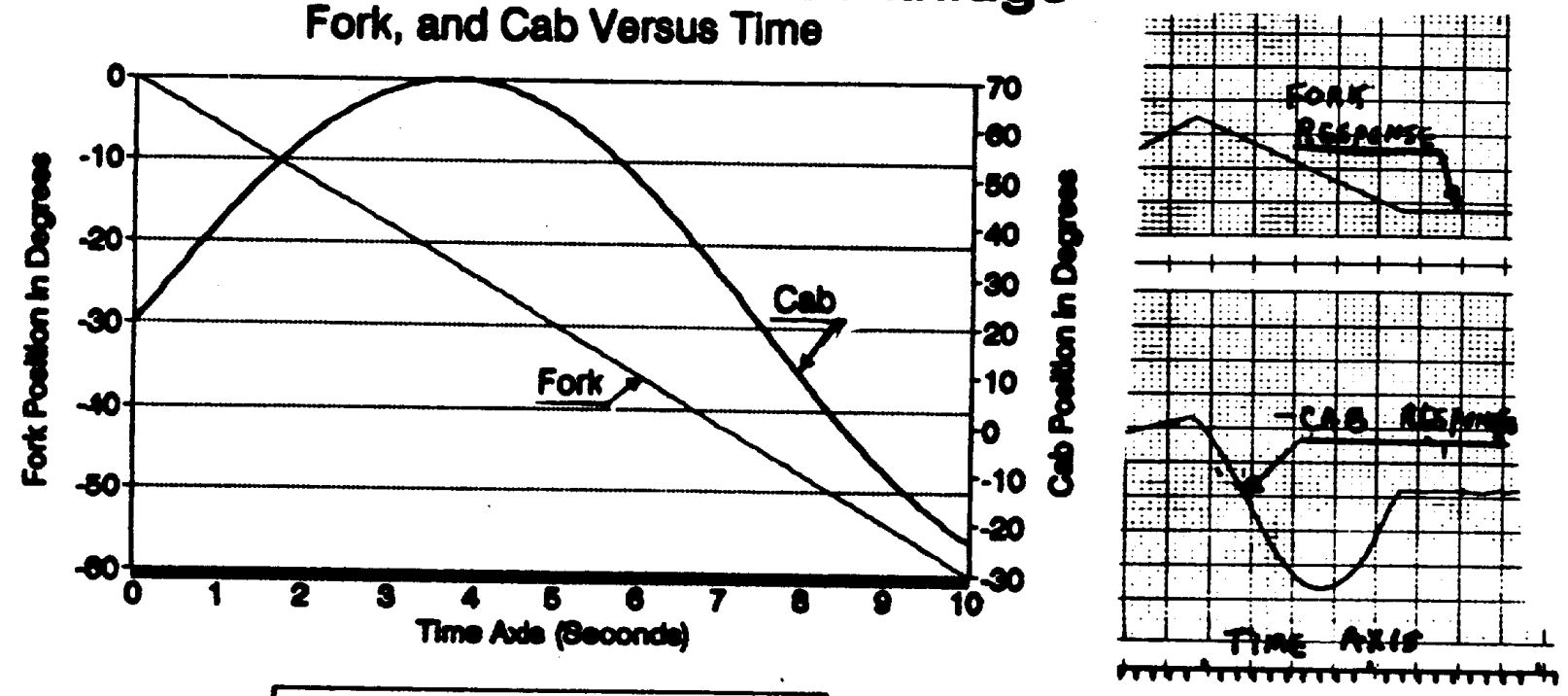

Figure (9) - Yo-Yo Turn For BFM (Basic Fighter Maneuver) 